Relations industrielles

Industrial Relations

\title{
Neill, Jon (sous la direction de), Poverty and Inequality: The Political Economy of Redistribution
}

\section{Ruth Rose}

Volume 54, numéro 1, 1999

Relations industrielles et nouveaux systèmes productifs

Industrial Relations in the New Workplace

URI : https://id.erudit.org/iderudit/051230ar

DOI : https://doi.org/10.7202/051230ar

Aller au sommaire du numéro

Éditeur(s)

Département des relations industrielles de l'Université Laval

ISSN

0034-379X (imprimé)

1703-8138 (numérique)

Découvrir la revue

Citer ce compte rendu

Rose, R. (1999). Compte rendu de [Neill, Jon (sous la direction de), Poverty and Inequality: The Political Economy of Redistribution]. Relations industrielles /

Industrial Relations, 54(1), 208-210. https://doi.org/10.7202/051230ar

Tous droits réservés (C) Département des relations industrielles de l'Université Laval, 1999
Ce document est protégé par la loi sur le droit d'auteur. L'utilisation des services d'Érudit (y compris la reproduction) est assujettie à sa politique d'utilisation que vous pouvez consulter en ligne.

https://apropos.erudit.org/fr/usagers/politique-dutilisation/ 


\section{Poverty and Inequality: The Political Economy of Redistribution}

publié sous la direction de Jon NEILL, Kalamazoo, Mich. : W. E. Upjohn Institute for Employment Research, 1997, 153 p., ISBN 0-88099-182-8 (alk. paper), ISBN 0-88099-181-X (pbk. : alk. paper).

En dépit d'une croissance économique impressionnante, l'incidence et le degré de la pauvreté ont augmenté de façon significative aux États-Unis depuis le début des années 1970, renversant une tendance à long terme. Ce phénomène a été accompagné d'un accroissement marqué des inégalités de revenu au détriment de $80 \%$ de la population. Cet ouvrage, dirigé par Jon Neill, découle d'une série de six conférences présentées à Western Michigan University en 1994-1995. Les objectifs étaient de mieux mesurer le phénomène d'appauvrissement, d'en cerner les racines et d'évaluer les politiques cherchant à le combattre, plus spécifiquement le Personal Responsibility and Work Opportunity Reconciliation Act adopté peu après la présentation des conférences.

Robert Haveman (Welfare Report-1996 Style) présente d'abord un bref historique des débats menant à la plus récente réforme de l'aide sociale. Imposée par la majorité républicaine, cette réforme limite de façon drastique l'accès à l'ADFC (Aid to families with dependant children principal programme d'assistance sociale aux États-Unis) : les immigrants, mêmes légaux, les mères adolescentes non mariées et les enfants nés d'une mère déjà bénéficiaire sont exclus et aucun bénéficiaire ne peut recevoir des prestations pendant plus de 60 mois au cours de sa vie. Selon Haveman, il n'y a aucune évidence que le système antérieur ait encouragé les prestataires à avoir plus d'enfants ou que la nouvelle loi sera plus efficace sur le plan administratif. Il questionne la légalité de l'exclusion des immigrants qui paient leur part des impôts et sont sujets à la conscription militaire. Prédisant une croissance future des dépenses liées à l'itinérance et le placement des enfants en foyer d'accueil, Haveman signale l'incapacité de la partie inférieure du marché du travail d'absorber 2 millions de travailleuses et travailleurs peu qualifiés et surtout de leur offrir des salaires permettant de sortir de la pauvreté. Haveman préconise des subventions salariales comme solution, mais demeure profondément pessimiste quant à la capacité des États-Unis de réduire la pauvreté de façon significative.

Rebecca M. Blank (Why Has Economic Growth Been Such an Ineffective Tool Against Poverty in Recent Years?) arrive à des conclusions similaires. Constatant que les personnes à faible scolarité participent au marché du travail pendant les reprises économiques, elle explique la croissance de la pauvreté entre 1969 et 1992 par une détérioration marquée des salaires versés aux hommes non détenteurs d'un diplōme universitaire, ainsi que par le fait que les femmes continuent de recevoir des piètres salaires en dépit d'un rétrécissement de l'écart avec les salaires masculins. Blank conclut que ni la croissance économique ni la disponibilité des emplois ne suffiront pour réduire la pauvreté à l'avenir. Elle, aussi, préconise comme solution diverses formes de suppléments au salaire, une meilleure perception des pensions alimentaires et des subventions aux frais de garde, mais souligne que ces mesures deviendront de plus en plus onéreuses à mesure que la population vulnérable à la pauvreté augmente.

John P. Formby (Regional Poverty and Inequality in the United States) présente et critique les concepts de base concernant les mesures de pauvreté et leur relation avec les mesures d'inégalité du revenu (mesures relatives et absolues de pauvreté, courbes de Lorenz, coefficients de Gini, etc.). Il préconise l'utilisation de l'index Sen qui tient compte non seulement du pourcentage de la population dont le revenu est inférieur au seuil de 
pauvreté, mais aussi de l'écart entre leur revenu et ce seuil, pondéré par le rang relatif de chacun parmi les pauvres. partir de six mesures de la pauvreté, Formby démontre que la pauvreté a augmenté entre 1979 et 1990 dans les quatre grandes régions des États-Unis. Lorsqu'on tient compte du revenu en espèces, on constate une croissance plus importante de la pauvreté et les rangs relatifs des régions changent, le nord-est étant plus pauvre relativement et l'ouest moins pauvre que ne l'indiquent les mesures conventionnelles. Sur le plan politique, Formby est surtout préoccupé du fait que les mesures de pauvreté basées sur le revenu monétaire ne montreront pas les effets des coupures des " coupons d'alimentation " (food stamps) récemment annoncées, alors que, dans la réalité, une partie importante de la population, incluant bon nombre d'enfants, deviendra plus pauvre.

Timothy M. Smeeding (The International Evidence on Income Distribution in Modern Economics) offre un portrait comparatif des inégalités du revenu et de leur évolution dans 25 pays, à partir des données du Luxembourg Income Study. Constatant un large éventail de résultats, il arrive, néanmoins, à la conclusion que le chômage et l'inégalité des revenus découlant du marché ont augmenté au cours des années 1980 dans presque tous les pays, particulièrement au Royaume-Uni, aux États-Unis, en Suède et en Finlande. Dans certains pays, notamment le Canada et la Finlande, les politiques sociales ont réussi à neutraliser ces augmentations, alors qu'aux ÉtatsUnis, au Royaume-Uni, en Suède et dans certains pays de l'Europe de l'Est, l'inégalité du revenu disponible (après impôts et transferts) a augmenté autant ou plus que celle du revenu de marché. Smeeding remarque particulièrement l'érosion de la classe moyenne au RoyaumeUni et aux États-Unis. Il est difficile de résumer toutes les informations qu'on peut tirer de ces comparaisons internationales, mais ce chapitre fournit une riche biblio- graphie permettant d'accéder au corpus croissant de recherche dans ce domaine.

Jere R. Behrman (From Parent to Child, Intergenerational Relations and Intrahousehold Allocations) examine d'abord la question de la transmission de la pauvreté d'une génération à l'autre. Il rapporte que la corrélation entre le revenu des parents et des enfants est d'environ $20 \%$ lorsqu'on utilise une seule année de données, ce qui suggère un degré de mobilité intergénérationnelle plutôt élevé. Toutefois, lorsqu'on utilise dix années de données sur le revenu, la corrélation augmente à $40 \%$. Behrman s'intéresse ensuite au degré de corrélation entre l'aptitude héritée par les enfants et l'investissement des parents dans le capital humain. Il soutient que les parents ne sont pas toujours capables d'investir suffisamment pour égaliser le taux marginal de rendement à l'éducation de leurs enfants et celui d'autres actifs, critère pour assurer que cet investissement est socialement efficace. Les parents américains ne semblent pas discriminer selon le sexe, mais tendent à favoriser les enfants les plus doués et les enfants les plus âgés, ce qui accroît la corrélation entre l'aptitude et le revenu viager. En Inde, par contre, les familles investissent davantage dans les garçons, particulièrement quand la nourriture se fait rare.

Le dernier texte de Gordon Tullock (The Reality of Redistribution) est, à mon avis, un simulacre de la recherche scientifique, s'appuyant sur une méthodologie tautologique qui permet à l'auteur de promouvoir ses biais idéologiques sans aucun fondement empirique ni même théorique. Tullock part de la prémisse "que l'idée qu'il existe un arbitrage entre la redistribution du revenu et de la production de la société et son taux de croissance est acceptée par tout le monde, malgré le fait qu'il existe peu d'évidence empirique " (p. 126, notre traduction). Le restant de l'exercice consiste à démontrer que les transferts redistributifs sont contre-productifs parce qu'ils 
ralentissent la croissance et donc réduisent le revenu futur, même des plus pauvres. De plus, en soulignant que la vaste majorité des peuples du monde sont beaucoup plus démunis que les Américains pauvres, il banalise la réalité de la pauvreté dans un pays qui prétend ētre un modèle d'égalité et de prospérité en affirmant qu'un "Américain résidant dans Harlem est davantage affecté par l'absence d'un téléviseur en couleur... qu'une mère du Sahel dont l'enfant meurt dans une famine qui prend les vies de plusieurs enfants du village " (p. 134, notre traduction). On croirait qu'une telle logique l'amènerait, au moins, à proposer que l'aide destinée aux Américains pauvres soit redirigée vers l'aide au développement international. Mais non, il croit que même là, il serait plus efficace de taxer les pauvres afin d'investir dans le capital qui assurerait une croissance à plus long terme.

Globalement, les six essais témoignent de la faillite des politiques américaines qui cherchent à intégrer les prestataires de l'aide sociale à un marché du travail caractérisé de plus en plus par des emplois précaires, à faible salaire et sans espoir d'avancement. Aucun des textes ne fait état de nouvelles recherches mais s'appuient sur des études déjà publiées ailleurs. Ce sont donc les bibliographies qui apportent le plus au lecteur.

Quoique le directeur, ainsi que les cinq premiers auteurs, semblent réellement préoccupés par les effets néfastes de la pauvreté et de l'incapacité de la croissance économique de renverser la tendance, ils sont tous plutôt fatalistes. Aucun n'essaie de tirer des leçons de la Grande Dépression des années 1930, une période où l'érosion des salaires a été aussi importante mais plus rapide. La restructuration fondamentale des relations économiques, notamment la reconnaissance du droit à la syndicalisation, et l'adoption des politiques sociales modernes ont été à la racine du déclin marqué de la pauvreté dans tous les pays occidentaux jusqu'aux années 1970. L'érosion actuelle des salaires, non seulement pour les personnes peu scolarisées, mais pour $80 \%$ de la population américaine, est-il le résultat d'un marché infaillible érigé en Dieu toutpuissant? Ou est-il le fait d'une petite élite de monopolistes qui, s'arrogeant de plus en plus de pouvoir économique et financier, se servent du slogan de "libre échange ", pour se libérer des contraintes des États interventionnistes et redistributifs?

RUTH ROSE

Université du Québec à Montréal 